\title{
Radiotherapy Equipment and Workforce in Turkey
}

\author{
(1) Hatice Bilge BECERIR, (1) Salih GÜRDALLI, ${ }^{2}$ (1) Bülent YAPICI,, (1) Fadime ALKAYA, 4 (1) Aydın ÇAKIR, ${ }^{5}$ \\ (1) Kadir YARAY, ${ }^{6}$ (1) Tamer Oğuz GÜRSOY7
}

\author{
'Department of Medical Physics, Istanbul University Institute of Oncology, Istanbul-Turkey \\ ${ }^{2}$ Department of Medical Physics, Bozlu Holding, Istanbul-Turkey \\ ${ }^{3}$ Department of Radiation Oncology, University of Acibadem Faculty of Medicine, Istanbul-Turkey \\ ${ }^{4}$ Department of Radiation Oncology, Istanbul Medicana International Hospital, Istanbul-Turkey \\ ${ }^{5}$ Istanbul Bilgi University, Vocational Faculty of Health Services, Istanbul-Turkey \\ ${ }^{6}$ Department of Radiation Oncology, University of Erciyes Faculty of Medicine, Kayseri-Turkey \\ ${ }^{7}$ Department of TUSEB (Presidency of Turkish Health Institutes), Health Institute of Turkey, Istanbul-Turkey
}

\section{OBJECTIVE}

Radiotherapy is a costly treatment that requires the proper use of human and financial resources. In any country, the radiation therapy equipment and workforce should be well planned for the appropriate use of resources and radiotherapy treatments. For proper planning, the current situation should be assessed and prepared for the targets to be achieved. This study aims to determine the current status of radiotherapy devices and the workforce in Turkey.
\end{abstract}

\section{METHODS}

Questions were sent to 141 centers to evaluate the number of devices, technical specifications, date of manufacture and the number of radiation oncologists, medical physicists, and radiotherapy technicians by Turkish Medical Physics Association. The responses from the centers were analyzed by region and compared with the situation in other countries.

\section{RESULTS}

The data collected in the study are from December 2019. The rate of radiotherapy devices in Turkey according to population is estimated to be 3.14 megavoltage (MV) external devices per 1 million people. The distribution is not homogeneous in all regions. The highest value is 4.59 in the Central Anatolia region, and the lowest is 0.78 in the Eastern Anatolia region. For each MV device, on average, there are 1.58 specialists in medical physics.

\section{CONCLUSION}

Our study has shown that Turkey has made significant progress in terms of radiotherapy equipment and workforce in the past decade. Nevertheless, although Turkey did not reach the standards of high-income European countries, it is comparable to middle-income countries.

Keywords: Radiotherapy equipment; Turkey; workforce.

Copyright $\odot$ 2021, Turkish Society for Radiation Oncology

\section{Introduction}

The application of evidence-based treatment protocols and new high-precision technology has strengthened the key role of radiotherapy in cancer management. According to available data, approximately half of all cancers are treated with radiotherapy.[1,2] In recent years, there has been a need to provide objective data to
Received: July 21, 2021

Accepted: August 03, 2021

Online: September 16, 2021

Accessible online at:

www.onkder.org

OPEN ACCESS This work is licensed under a Creative Commons

Attribution-NonCommercial 4.0 International License.
Dr. Hatice Bilge BECERIR

İstanbul Üniversitesi Onkoloji Enstitüsü,

Medikal Fizik Anabilim Dalı,

İstanbul-Turkey

E-mail: hbilge@istanbul.edu.tr 
support the critical role and position of radiation therapy in the face of the rapidly changing technological advancement of radiotherapy.

Since 1982, the European Organization for Research and Treatment of Cancer-Radiation Oncology Group (EORTC-ROG) has launched a quality assurance program to establish standards for the participation of member centers in clinical trials involving radiotherapy and to check the consistency of data. In 1989, EORTC-ROG created a survey and developed it over the years. Between December 2005 and October 2007, 98 active EORTC-ROG member organizations from 19 countries completed the report online and provided survey responses. According to the results of this study, a total of 197.000 patients (an average of 2016 patients per center) were treated in 98 centers per year. The number of patients per radiation oncologist, radiation physicist, and radiotherapy technician was 258,420 , and 107 , respectively. The average annual number of patients per treatment device was 488 and 1117 for a CT simulator/classic simulator.[3]

The project "Radiation Therapy for Cancer" was launched in 2003 by the European Society for Radiotherapy-Quantitation of Radiation Therapy Infrastructure and Staffing (ESTRO). The aim of the project was to gather information and to provide general guidelines for infrastructure and personnel across Europe. In this study, countries were divided into three groups as low $(<\$ 3000)$, medium ( $\$ 3000-\$ 10.000)$, and high (more than $\$ 10,000$ ) according to their annual national income levels. Survey questions were sent to all countries, and the responses were evaluated according to the income groups. Forty-one countries (93.2\%), representing 99\% of the European population, responded. The principles of the linear accelerator and the number of staff were present in approximately $40 \%$ of the countries. [4,5]

Today, there is a need for objective data on cost and cost-effectiveness against ever-increasing maintenance costs. For this reason, ESTRO launched the Health Economics in Radiation Oncology (HERO) project to develop a knowledge base model for health and economic evaluation of radiation treatments at the European level.[5-7]

Although it is easy to obtain statistical information in countries with high annual income, for example, Europe, it is not easy in low- and medium-income countries. Grover et al.[8] tried to create a model for radiotherapy equipment by evaluating 49 articles on the subject to reveal the status of radiotherapy facilities in countries with low and medium annual national income. The authors divided the countries into four regions: Africa, Asia,
East Europe, and South America. The distribution of radiotherapy devices is very heterogeneous, as can be expected. For example, while $60 \%$ of radiotherapy devices are found in Egypt, very few of them are in other African countries, and their age is over 20 years. $[9,10]$ In this article, the data that we used for comparison are from the paper published in by Göksel et al.[11]

The current status of radiotherapy facilities, the diversity of devices, the number of medical physicists, radiation oncologists and radiotherapy technicians, and their comparison with the situation in other countries are essential for establishing plans for the future. This study by the Turkish Medical Physics Association aimed to determine the number of devices in radiotherapy, their technical characteristics, and the workforce in radiotherapy as well as to compare the current status of Turkey with the situation in other countries of the world.

\section{Materials and Methods}

The Turkish Medical Physics Association sent online questionnaires by e-mails to the responsible medical physicists at 141 radiotherapy centers to evaluate the technical characteristics of the devices, the year it was established, and the number of medical physicists, radiation oncologists, and radiotherapy technicians. The number of radiotherapy centers is taken from the website of the Turkish Association of Radiation Oncology. The responsible physicists of the centers were contacted by phone and answering the questionnaires was encouraged. All centers responded, and the data were analyzed. The responses were divided into seven regions, and the first six most densely populated cities were evaluated. Information about the population was obtained from data from the Turkish Ministry of Health. The data collected in the study are from December 2019.

Turkish population is 83.154 million by December 2019. The incidence of cancer will be 3-3.2 per 1000 people, which means that there will be 245.000-265.000 new cancer patients.[10] Radiotherapy is applied to $60 \%$ of cancer patients, and approximately $25 \%$ of these patients must be treated again due to metastasis and recurrence. This issue indicates that the number of patients requiring radiotherapy treatment is expected to be $170.000-185.000$ per annum.[11-15]

The number of centers $(\mathrm{CN})$, linear accelerators (LA), TomoTherapy systems (TT), CyberKnife systems (CK), GammaKnife systems (GK), intraoperative devices (IORT), brachytherapy devices (BRT), and CT simulators in Turkey were determined by region. Megavoltage (MV) devices include LA, TT, CK, and GK, 
do not include IORT and BRT devices. The number of MV devices per 1 million population and the number of $1 \mathrm{MV}$ devices for 450 new patients were determined. The number of radiation oncologists, medical physicists, and radiotherapy technicians in radiotherapy were determined and compared with results from the international literature.

\section{Results}

There are 141 radiotherapy centers in Turkey; the number of LA, TT, CK, GK, IORT, and BRT devices, CT simulators, and MV devices per 1 million population $(\mathrm{MV} / 1 \mathrm{M})$ are shown in Table 1 . There is one Cobalt-60 devices in Turkey. When calculating MV devices/1M, external devices such as LA, TT, CK, and GK systems were included in the calculation parameters; however, IORT and BRT devices were not included.

In radiotherapy, radiation oncologists, medical physicists, and radiotherapy technicians constitute the essential workforce; the summary for Turkey is shown in Table 2.

Table 3 shows the available radiotherapy workforce in Turkey in 2019, which is in line with the answers from the centers. The distribution of devices by year of production is shown in Figure 1. The number of LA, radiation oncologists, medical physicists, and radiotherapy technicians per 1 million people in Turkey in December 2019 are shown in Figure 2.

\section{Discussion}

The proper planning of radiotherapy equipment and workforce is necessary for appropriate radiotherapy treatment in any country. There are multiple methods to determine the required radiotherapy equipment and workforce. The most important method is IAEA recommendation of $4 \mathrm{MV}$ devices per 1 million people. [16] Turkey has a population of $83.154,997$ according to data from the Turkey Ministry of Interior. According to the IAEA approach, in Turkey, the average is 3.14 MV devices per 1 million people. In the Mediterranean, East Anatolia, Aegean, Southeast Anatolia, Central Anatolia, Black Sea, and Marmara regions, this average is $3.01,1.82,2.64,0.78,4.59,2.01$, and $4.13 \mathrm{MV}$ devices, respectively. The Central Anatolia region has the highest rate of 4.59 MV devices, while the Southeast Anatolia region has the lowest rate of $0.78 \mathrm{MV}$ devices. Considering radiotherapy device dispersion worldwide, 26\% of MV devices are in North America, $34 \%$ are in Europe, and $40 \%$ are in other countries. Per 1 million population, European countries have 6 MV devices, African countries have $1 \mathrm{MV}$ device, and low- and middle-income Asian countries have $2 \mathrm{MV}$ devices.[2] The IAEA survey results showed that the number of MV devices per million people in postSoviet Union countries is between 0.1 and 3.5.[16]

The first study on radiotherapy equipment and workforce in Turkey was conducted in 2004 by Seyfettin Kuter. According to their results, in 2002, the CN was 50 , the number of LA was 40 , the number of Co60 devices was 48 , the number of BRT was 19 , and the number of simulators was 53. Besides, there were 351 radiation oncologists, 98 medical physicists, and 271 radiotherapy technicians.[13] The first study to determine the status of radiotherapy devices in Turkey and to provide a forward-looking perspective was carried out by Göksel et al.[11] The information in this study

Table 1 Distribution of the radiotherapy devices, according to the region of Turkey

\begin{tabular}{|c|c|c|c|c|c|c|c|c|c|c|}
\hline Regions & CN & LA & TT & CK & GK & BRT & IORT & $\mathbf{C T}$ & Population & $\begin{array}{c}\text { MV/1M } \\
\text { (except BRT } \\
\text { and IORT) }\end{array}$ \\
\hline Mediterranean & 19 & 27 & 4 & - & 1 & 6 & - & 20 & $10.627,530$ & 3.01 \\
\hline East Anatolia & 8 & 9 & 2 & - & - & - & - & 7 & $6.045,786$ & 1.82 \\
\hline Aegean & 18 & 23 & 3 & 1 & 1 & 5 & - & 21 & $10.618,433$ & 2.64 \\
\hline Southeast Anatolia & 7 & 6 & 1 & - & - & 1 & - & 6 & $8.975,618$ & 0.78 \\
\hline Central Anatolia & 28 & 54 & 2 & 3 & 2 & 5 & 1 & 28 & $13.273,751$ & 4.59 \\
\hline Black Sea & 10 & 14 & 1 & 1 & - & 1 & - & 10 & $7.963,476$ & 2.01 \\
\hline Marmara & 51 & 83 & 9 & 7 & 7 & 16 & 5 & 55 & $25.650,403$ & 4.13 \\
\hline Total & 141 & 216 & 22 & 12 & 11 & 35 & 6 & 147 & $83.154,997$ & 3.14 \\
\hline
\end{tabular}

CN: Number of centers; LA: Number of linear accelerators; TT: Number of TomoTherapy systems; CK: Number of CyberKnife systems; GK: Number of GammaKnife systems; IORT: Number of intraoperative radiotherapy units; BRT: Number of brachytherapy units; CT: Number of computerized simulators; MR: Magnetic resonance; MV: Megavoltage 
Table 2 Number of medical physicists present and required for radiotherapy in Turkey. The number of medical physicians required was calculated based on the expectation of 170.000-185.000 cancer patients likely to receive radiotherapy/year[10]

\begin{tabular}{|c|c|c|c|c|c|c|}
\hline Regions & $\mathrm{CN}$ & MV & MP & $\begin{array}{c}\text { MP/MV } \\
\text { (except BRT) }\end{array}$ & $\begin{array}{c}\text { MP/400 } \\
\text { patients } \\
\text { (suggested) }\end{array}$ & $\begin{array}{c}\text { MV/450 } \\
\text { patients } \\
\text { (suggested) }\end{array}$ \\
\hline Mediterranean & 19 & 32 & 47 & 1.47 & $54-59$ & $48-53$ \\
\hline East Anatolia & 8 & 11 & 18 & 1.63 & $30-33$ & $27-30$ \\
\hline Aegean & 18 & 28 & 55 & 1.96 & $53-58$ & $47-52$ \\
\hline Southeastern Anatolia & 7 & 7 & 18 & 2.57 & $46-50$ & $41-44$ \\
\hline Central Anatolia & 28 & $61+C 060$ & 99 & 1.6 & $65-71$ & $58-63$ \\
\hline Black Sea & 10 & 16 & 23 & 1.43 & $39-43$ & $35-38$ \\
\hline Marmara & 51 & 106 & 155 & 1.46 & $126-137$ & $112-122$ \\
\hline Total & 141 & 262 & 415 & 1.58 & $425-462$ & $378-411$ \\
\hline
\end{tabular}

CN: Number of centers; MV: Megavoltage; MP: Medical physicist; BRT: Number of brachytherapy units; Co-60: Cobalt-60

Table 3 Radiation oncology workforce in Turkey, December 2019. The numbers of radiation oncologists were taken from the Turkish radiation oncology association and the numbers of radiotherapy technicians from the association of radiotherapy technicians

\begin{tabular}{lcc} 
Staff & Number (2019) & Suggested \\
\hline Radiation oncologist & 596 & $680-740(1 \mathrm{RO} / 250 \mathrm{patients})$ \\
Medical physicist & 415 & $425-462(1 \mathrm{MP} / 400 \mathrm{patients})$ \\
Radiotherapy technician & 1100 & $1700-1850(1 \mathrm{RTT} / 100 \mathrm{patients})$ \\
\hline
\end{tabular}

RO: Radiation oncologist; MP: Medical physicist; RTT: Radiotherapy technician

was obtained through a survey and has been confirmed by IAEA data. According to the data from this study, in 2010, there were 1.8 MV devices per 1 million people in Turkey. According to the study by the Turkish Medical Physics Association in 2019, there are 262 MV devices (216 LAs include $1 \mathrm{MR}$ linear accelerator, 1 device, and 22 TT, 12 CK, 11 GK) and 6 IORT devices in Turkey.

Although the number of radiotherapy devices has increased significantly over the past 8 years, it has only achieved the criterion recommended by IAEA in Central Anatolia and the Marmara Region. The numbers for the other five regions do not meet this criterion and are far from the average of $6 \mathrm{MV}$ devices per 1 million population in high-income countries. Istanbul, Ankara, Izmir, Bursa, Antalya, and Adana are the top six most populous cities in Turkey by constituting $37 \%$ of the population; there are 5.1, 7.07, 4.12, 4.25, 3.58, and 4.91 MV devices per 1 million populations, respectively, in these cities. About $53 \%$ of the radiotherapy centers, $62.2 \%$ of the LA, and almost all of the special devices (TT, CK, GK, and IORT) are located in these cities.

The population is not the only method that determines the standards for radiotherapy. There is also a method that determines the need considering annual cancer patients. According to this reference, one MV device is required for every 200-500 new radiotherapy patients.[3] EORTC ROG has adopted the requirement of one MV device for every 450 new patients and recommends that the number of treated patients for one MV device does not exceed 600.[3] Turkish population is 83.154 million by December 2019, according to EORTC-ROG recommendation; Turkey needs 377-411 MV devices. Current device number of Turkey is far from the EORTC-ROG recommendation. Radiotherapy centers should not work with only a single device and should start serving with at least two devices. According to IAEA device criteria, there should be 332 external radiotherapy devices in Turkey. The inadequate number of devices can cause treatment interruptions in the event of device failures. It is recommended that the devices used in radiotherapy be $<10$ years old.[3] In Turkey, 44 LAs were taken in 2009 and in previous years. These devices will need to be replaced in the near future. In addition, the total number of 35 BRT has not changed since 2010. New techniques developed in external radiotherapy might be the reason for the number is not increasing. 
Distribution of radiotherapy devices by years in Turkey

- $\mathrm{LA}=\mathrm{TT} \approx \mathrm{CK}=\mathrm{GK} \approx \mathrm{BRT}$

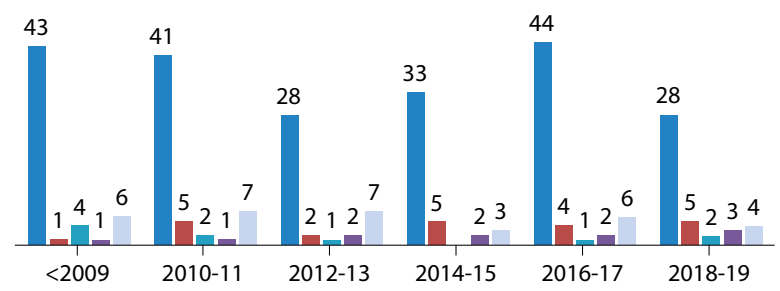

Fig. 1. Distribution of the years in which linear accelerators in Turkey were produced.

LA: Number of linear accelerators; TT: Number of TomoTherapy systems; CK: Number of CyberKnife systems; GK: Number of GammaKnife systems; BRT: Number of brachytherapy units.

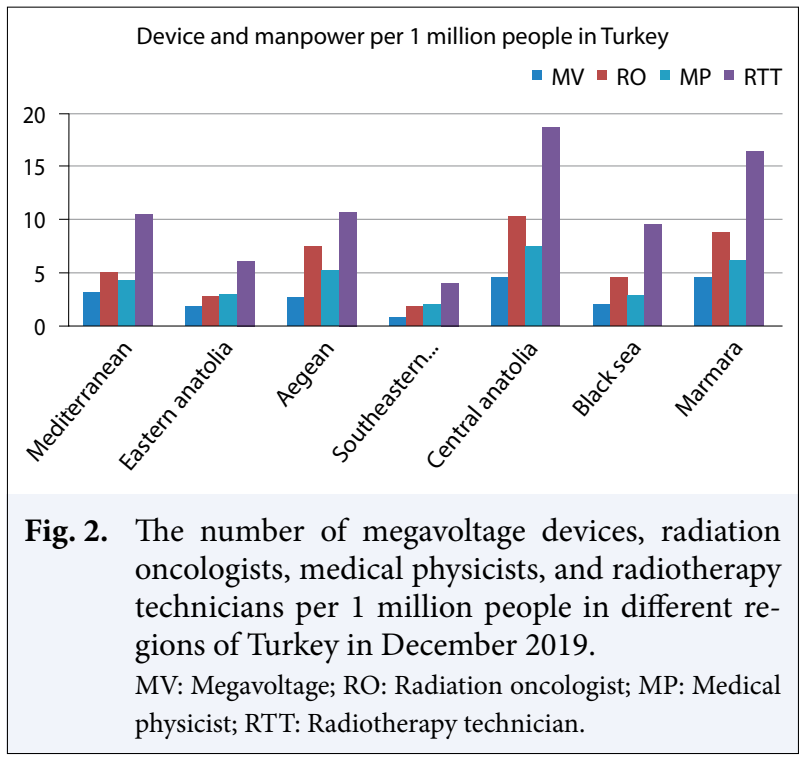

Accurate determination of the target volumes and critical structures is the first step for successful radiotherapy. This is only possible when simulation is performed with 3D CT. There are $147 \mathrm{CT}$ simulation units in Turkey. One CT simulator for each center was recommended by the IAEA and ESTRO guidelines. However, centers with three or more LAs are recommended to have two CT simulators. In Turkey, the number of CT simulators is sufficient. The number of patients should not exceed 2400 for one CT simulator in a year. $[15,16]$ In case of exceeding the recommended number of patients for one CT simulator, it has been suggested to use the second CT simulator.

In radiotherapy, radiation oncologists, medical physicists, and radiotherapy technicians are the essential workforce. While determining the need for radio- therapy devices and the required manpower, there are different methods determined by taking into account parameters such as population, number of patients, and number of fractions.

\section{Radiation Oncologists}

There is one radiation oncologist for every $150-400$ radiotherapy patients in the world. The range is very wide. One radiation oncologist per 250 radiotherapy patients on average is the accepted criterion. $[6,7,15,17]$ Turkey has adopted 250 patients per radiation oncologist. Accordingly, there should be 680-740 radiation oncologists. At present, the number of radiation oncologists is 596 in Turkey. The number of radiation oncologists may vary according to their specialty in their country. In some countries, radiotherapy and chemotherapy are performed by the same doctor.

\section{Radiotherapy Technicians}

There must be a minimum of two radiotherapy technicians for one device. This number may vary on the defined workload; it should be considered that the procedures for some techniques such as IGRT increase the workload by repetitive steps of patient setups. There were 600 radiotherapy technicians in Turkey in 2010. [11] The number was raised to 1100 , according to the data collected in 2019. The number of radiotherapy technicians in operation seems to be less than necessary. As stated in the ESTRO-HERO study, the number of radiotherapy technicians is higher in European countries than in our study. $[6,7,15,17]$

\section{Medical Physicists}

In Turkey, the number of medical physicists working in radiotherapy is 415 . EFOMP recommends that a medical physicist serves 400-750 patients annually and that an average of 400 patients is eligible. A total of 425-462 medical physicists are needed for 170.000-185.000 new radiotherapy patients in Turkey. Considering that there are no assisting occupational groups planning treatment such as dosimetrists and technologists in Turkey, this number should be more than recommended. The recommendation of two medical physicists per linear accelerator is also widely accepted. In Turkey, the number of medical physicists per MV devices is 1.58.

The Institute of Physics and Engineering in Medicine published a report in 2002 that made recommendations to determine the minimum number of medical physicists in the field of radiotherapy.[15] According to this report, the number of physicists required depends on the complexity of the device and treatments 
and the operation of the department. It is a correct approach as the planning time of patients varies with the techniques chosen. Furthermore, patient and linear accelerator-based quality control procedures should be taken into account. Medical physicists are also responsible for radiation safety, medical physics education in the department, and the department quality processes. It is not realistic to determine the number of medical physicists based only on the number of patients or the number of LA.

A medical physicist called a health physicist by the government in Turkey. According to law, a health physicist is defined as a person who has a master's degree in one of the fields of radiotherapy, nuclear medicine, or radiology after graduation. To become a medical physicist, he/she is required to have a bachelor's degree in physics, a graduate degree in physics engineering and nuclear energy engineering, and a master's degree in health physics. There are many health physics graduate programs in Turkey. Some of them are in institutions where there are no radiotherapy centers. These students graduate by only taking theoretical courses. Nothing prevents graduate programs from opening in universities without clinical equipment. Students who graduate from some programs can thus graduate without the necessary practical training. In addition, it is not recommended for a newly graduated person to start working alone without the necessary experience with an experienced specialist. EFOMP recommends that a recently graduated medical physicist should work with an experienced medical physicist for 5 years. While trying to reach the required number of occupational groups in radiotherapy, the quality of vocational training should not be ignored. Unfortunately, the educational quality of master's programs in practical application is not consistent in Turkey.

The ESTRO-HERO study revealed that the economic conditions of countries did not affect the number of radiation oncologists but did affect the number of medical physicists and radiotherapy technicians. [7] In low-income countries, the number of medical physicists and technicians is lower than that in highincome countries.

\section{Conclusion}

Radiotherapy is a costly treatment that requires the proper use of the workforce and financial resources. Radiotherapy-related international organizations have established guidelines for determining infrastructure and human needs, recommending one linear acceler- ator for 450 patients per year, one radiation oncologist for 200-250 patients per year, and one medical physicist for 450-500 patients per year. Considering these suggestions, Turkey has made significant progress in recent years. The situation in Turkey is better than that in middle-income countries but not high-income countries. However, it should be noted that these are merely rough rules, and the requirements mainly depend on the population structure, the incidence of cancer, and treatment strategies, which differ in various countries.

Peer-review: Externally peer-reviewed.

Conflict of Interest: All authors declared no conflict of interest.

Ethics Committee Approval: The decision was made in the board of directors of the National Medical Physics Association (No: 269, Date: 08/12/21). No biometric and biological materials belonging to humans and experimental animals were used in the study.

Financial Support: None declared.

Authorship contributions: Concept - H.B.B., S.G., B.Y.; Design - S.G., B.Y.; Supervision - H.B.B., S.G.; Funding S.G., B.Y.; Materials - None; Data collection and/or processing - A.Ç., F.A.; Data analysis and/or interpretation - K.Y., T.O.G., S.G.; Literature search - F.A., K.Y.; Writing - H.B.B., S.G.; Critical review - H.B.B., S.G.

\section{References}

1. Delaney GP, Jacob S, Featherstone C, Barton MB. The role of radiotherapy in Cancer care: Estimating optimal utilization from a review of evidence based clinical guide lines. Cancer 2005;104(6):1129-37.

2. Barton MB, Jacob S, Shafiq J, Wong K, Thompson SR, Hanna TP, et al. Estimating the demand for radiotherapy from the evidence: A review of changes from 2003 to 2012. Radiother Oncol 2014;112(1):140-4.

3. Budiharto T, Musat E, Poortmans P, Hurkmans C, Monti A, Bar-Deroma R, et al. Profile of European radiotherapy departments' contributing to the EORTC Radiation Oncology Group (ROG) in the $21^{\text {st }}$ century. Radiother Oncol 2008;88(3):403-10.

4. Slotman B, Cottier B, Bentzen S, Heeren G, Lievens Y, van den Bogaert W. Overview of national guidelines forInfra structure and staffing of radiotherapy. ESTRO-QUARTS: Work package 1. Radiother Oncol 2005;75(3):349-54.

5. Lievens Y, Grau C. Health economics in radiation oncology: Introducing the ESTRO HERO project. Radiother Oncol 2012;103(1):109-12.

6. Dunscombe P, Grau C, Defourny N, Malicki J, Bor- 
ras JM, Coffey M, et al. Guidelinesfor equipment and staffing of radiotherapyfacilities in theEuropeancountries: Final results of the ESTRO-HERO survey. Radiother Oncol 2014;112(2):165-77.

7. Lievens Y, Defourny N, Coffey M, Borras JM, Dunscombe P, Slotman B, et al. Radiotherapy staffing in the European countries: Final results from the ESTROHERO survey. Radiother Oncol 2014;112(2):178-86.

8. Grover S, Xu MJ, Yeager A, Rosman L, Groen R, Chackungal $\mathrm{S}$, et al. A systematic review of radiotherapy capacity in low and middle income countries. Front Oncol 2015;4:380.

9. Abdel-Wahab M, Bourgue J, Pynde Y, Izewsk J, Van der Merwe D, Zubzarreta E, et al. Status of radiotherapy resources in Africa: An international atomic energy agency analysis. Lancet Oncol 2013;14(4):e16875.

10. Levin CV, El Gueddari B, Meghzifene A. Radiation therapy in Africa distribution and equipment. Radiother Oncol 1999;52(1):79-83.

11. Göksel F, Koc O, Ozgul N. Gultekin M, Abacioglu $M$, Tuncer $M$, et al. Radiation oncology facilities in Turkey: Current status and future perspectives. Asian
Pac J Cancer Prev 2011;12(9):2157-62.

12. Kuter S, Cakır A. Survey of radiation oncology centers, equipment and manpower. Turk J Oncol 2004;19(3):84-96.

13. Karakaya MD. Provincial and Regional Population Projections for the Centenary of the Republic of Turkey, Dissertation. Ankara: Hacettepe University; 2009. p. 416.

14. Turkish Ministry of Health. Health Care Services that Require Specific Planning in Turkey. Vol. 836. Turkish Ministry of Health; 2011. p. 1011-1023

15. Institute of Physics and Engineering in Medicine. Guidelines for the Provision of a Physics Service to Radiotherapy. London: Institute of Physics and Engineering in Medicine; 2002.

16. Rosenblatt E, Fidarova E, Ghosh S, Zubizarreta E, Unterkirhere O, Semikoz N, et al. Quality of radiotherapy services in post-Soviet countries: An IAEA survey. Radiother Oncol 2018;127(2):171-7.

17. International Atomic Energy Agency. Planning National Radiotherapy Services: A Practical Tool. IAEA Human Health Series No. 14. Vienna: International Atomic Energy Agency; 2010. 